\title{
Influence of medium composition on secondary metabolites synthesis in Drosera binata
}

\author{
Milena Chraniuk, Kinga Siatkowska, Rafał Banasiuk \\ Institute of Biotechnology and Molecular Medicine, Trzy Lipy 3, Gdańsk, Poland \\ Corresponding author: milena.chraniuk@herbiopharm.pl \\ DOI: $10.31708 /$ spi3.2018/chra.cns18
}

\section{Introduction}

Plant secondary metabolites are used in medicine and industry. Their larger amounts can be received by the plant in vitro cultures. Appropriate plant growth factors promote increased synthesis of biologically active compounds which can be extracted and examined in numerous biological activity tests. The main active compound for Droseraceae is plumbagin. Biosynthesis of this compound and other secondary metabolites depends on many factors such as growth medium $\mathrm{pH}$ and osmotic pressure. This work aims to test plants' growth conditions and to analyze quantitatively and qualitatively Drosera binata ethanolic extracts.

\section{Materials and Methods}

Drosera binata from University of Gdansk plant cultures, MS salts (Duchefa Biochemie, Netherlands), plant agar (Duchefa Bioechemie, Netherlands), sucrose (Acros, Belgium), C18 column modified with phenyl groups Zorbax SB-Phenyl 3,5 $\mu \mathrm{m}, 4,6 \mathrm{~mm} \times 150 \mathrm{~mm}$ (Agilent, USA), ethanol (Avantor, Poland), acetonitrile HPLC grade (Avantor, Poland), trifluoroacetic acid (Alfa Aesar, USA), deionized water. Plant cultures - $D$. binata plants were cultivated on solid MS medium with a variable amount of macroelements (3/4 MS, 1/2 MS, 1/3 MS) with different $\mathrm{pH}(5.2 ; 5.5 ; 6)$. Mediums were supplemented by $1 \%, 2 \%$ or $3 \%$ of sucrose. Cultures were maintained for six weeks. After the growth period, the material was collected, cleaned from medium and stored at $-20^{\circ} \mathrm{C}$. Extraction -1 $\mathrm{mg}$ of frozen plant material was extracted three times with $1 \mathrm{ml}$ of $95 \%$ ethanol for $48 \mathrm{~h}$ at room temperature. After this time, extracts were collected and kept in $-20^{\circ} \mathrm{C}$ until HPLC analysis. Extracts were analyzed with use of HPLC system Ultimate 3000 (Dionex, USA) with a C18 column modified with phenyl groups, UV-VIS detector at wavelength $254 \mathrm{~nm}$ and gradient elution program.

\section{Results}

The highest concentrations of secondary metabolites were obtained for $1 / 3 \mathrm{MS}$ medium at $\mathrm{pH} 6$ with the addition of $3 \%$ sucrose. The lowest concentrations of secondary metabolites were obtained for $3 / 4 \mathrm{MS}$ at $\mathrm{pH}$ 5.2 and $3 \%$ sucrose and $3 / 4 \mathrm{MS}$ at $\mathrm{pH} 5.5$ and $1 \%$ sucrose. Metabolite with the highest biosynthesis level was plumbagin (concentration ranges $0,17 \mathrm{mg} / \mathrm{ml}-0,44$ $\mathrm{mg} / \mathrm{ml}$ ). Quercetin has the highest ability to extract from the analyzed plant material. Concentrations of the compound in the first extract are about ten times higher than concentrations in the second extract. Quercetin concentrations in the second and third extract are similar. The lowest level of extraction has droserone, concentration ratio for the first and second extracts is 1.75 and for second and third extracts 1.36.

\section{Discussion}

The concentration of plumbagin synthesized in 6 weeks by $D$. binata reached half values of the concentrations obtained for $D$. rotundifolia and $D$. intermedia in growth time from 6 to 24 months (Baranyai, Bäcker, Reich, \& Lindequist, 2016). Analyzed in this study secondary metabolites are also synthesized by other Drosera spp. (Marczak, Kawiak, Łojkowska, \& Stobiecki, 2005). In pharmaceutical production, e.g. D. capensis can be a source of 7-methyljuglone and $D$. binata can be used as a good natural source of plumbagin (Samaj et al., 1999). Plant extracts are rich in biologically active compounds, and they have a great antimicrobial potential (Krolicka et al., 2008). They can be used in mixtures with other substances as an antimicrobial agent (Krychowiak et al., 2014) or natural feed additive that supports the development of farm animals beneficial microflora (Elghandour et al., 2018).

\section{Acknowledgements}

Research financed from project funds implemented under measure 1.1 "R\&D projects of enterprises", sub-measure 1.1.1 "Industrial research and development carried out by enterprises" of the Intelligent Development Operational Program 2014-2020, co-financed by the European Regional Development Fund POIR.01.01.01-0000739/16.

\section{References}

Baranyai, B., Bäcker, C., Reich, C., \& Lindequist, U. (2016). The production of 7-methyljuglone, plumbagin and quercetin in wild and cultivated Drosera rotundifolia and Drosera intermedia. Mires and Peat, 18(19), 1-8. https://doi.org/10.19189/MaP.2016.OMB.228 
Elghandour, M. M. M. Y., Kanth Reddy, P. R., Salem, A. Z. M., Ranga Reddy, P. P., Hyder, I., BarbabosaPliego, A., \& Yasaswini, D. (2018). Plant Bioactives and Extracts as Feed Additives in Horse Nutrition. Journal of Equine Veterinary Science, 69, 66-77. https://doi.org/10.1016/j.jevs.2018.06.004

Krolicka, A., Szpitter, A., Maciag, M., Biskup, E., Gilgenast, E., Romanik, G., ... Lojkowska, E. (2008). Antibacterial and antioxidant activity of the secondary metabolites from in vitro cultures of Drosera aliciae. Biotechnology and Applied Biochemistry, 184, 175-184. https://doi.org/10.1042/BA20080088

Krychowiak, M., Grinholc, M., Banasiuk, R., KrauzeBaranowska, M., Głód, D., Kawiak, A., \& Królicka, A. (2014). Combination of silver nanoparticles and Drosera binata extract as a possible alternative for antibiotic treatment of burn wound infections caused by resistant Staphylococcus aureus. PLOS ONE, 9(12), e115727.

https://doi.org/10.1371/journal.pone.0115727

Marczak, Kawiak, A., Łojkowska, E., \& Stobiecki, M. (2005). Secondary metabolites in in vitro cultured plants of the genus Drosera. Phytochemical Analysis, 16(3), 143-149.

https://doi.org/10.1002/pca.833

Samaj, J., Blehova, A., Repcak, M., Ovecka, M., Bobák, M., Šamaj, J., ... Bobák, M. (1999). Drosera Species (Sundew): In Vitro Culture and the Production of Plumbagin and Other Secondary Metabolites. Medicinal and Aromatic Plants, 11, 105. https://doi.org/10.1007/978-3-662-08614-8_7 